\title{
Ultrasound in the early diagnosis of heterotopic ossification in patients with spinal injuries*
}

\author{
R Bodley BM BCh FRCR FRACGP, A Jamous MD, MSc(Oxon), D Short MBBs \\ MRCP
}

Department of Radiology \& National Spinal Injuries Centre, Stoke Mandeville Hospital, Aylesbury, Bucks HP21 8AL, England.

Heterotopic ossification ( $\mathrm{HO}$ ) is a potentially disabling complication of spinal injuries and other chronic disorders. It is of unknown aetiology and currently there is no easy or convenient diagnostic method that will allow very early confirmation of the inflammatory changes that precede osteoid and, later, true bone formation. Clinical experience, however, indicates that early treatment with radiotherapy, antiinflammatory agents or diphosphonates is needed to control the progression.

This study was undertaken to assess the role of ultrasound (US) in the very early diagnosis of $\mathrm{HO}$ in patients with spinal injuries.

US was found to be very sensitive in detecting focal soft tissue abnormalities around joints and in the muscles of these patients. If combined with a Doppler study to exclude deep venous thrombosis (DVT), and infection or tumour could be excluded clinically, US was extremely accurate in predicting the presence or absence of early $\mathrm{HO}$ changes within hours of the clinical manifestation. In 2 patients it successfully predicted $\mathrm{HO}$ in the opposite leg before clinical signs were evident.

This study also provided supportive evidence of the theory of microtrauma in the aetiology of $\mathrm{HO}$.

As ultrasound is portable, safe, cheap, reproducible and accurate, it is the method of choice in the early diagnosis of HO. It allows early treatment to prevent the formation of osteoid and subsequent bone formation.

Keywords: ultrasound; heterotopic ossification; early diagnosis; spinal cord injuries.

\section{Introduction}

Heterotopic ossification (HO), also known as paraarticular ossification (PAO), ectopic ossification (EO) and periarticular new bone formation (PNBF) is a well known complication of several conditions, notably spinal injuries, head injuries, tetanus and severe burns as well as occurring after hip and rarely knee joint replacement surgery. ${ }^{1-3}$ It is a potentially disabling affliction

*The bulk of this study was presented at IMSOP, Rome, 1989 and the whole study was presented at AFIGAP, Mulhouse, May 1992. of unknown aetiology in which bone is formed in the soft tissues around the large joints below the level of injury with consequent decreased function and mobility, occasionally complete ankylosis, and an increased risk of pressure sores. ${ }^{4}$

Currently, the most commonly used early diagnostic technique is the three phase radioisotope bone $\operatorname{scan}^{5-7}$ but this requires expensive specialist equipment, employs ionising radiation, is relatively nonspecific and does not exclude the main differential diagnosis, deep venous thrombosis (DVT), so that other studies are needed. ${ }^{8}$ 
Ultrasound is well established as an accurate assessment technique for muscles and other soft tissues ${ }^{9-11}$ and is increasingly becoming the method of choice for the diagnosis of deep venous thrombosis. ${ }^{12.13}$ This study was undertaken to assess the role of ultrasound in the early diagnosis of $\mathrm{HO}$. The initial study was undertaken in 1987-1989 with a further study in 1992 using Doppler ultrasound for venous assessment.

\section{Methods}

Ultrasound examination using $3.5 \mathrm{MHz}$, $5 \mathrm{MHz}$ and $7.5 \mathrm{MHz}$ real times probes (Hitachi EUB 325 or Toshiba SSA 250A) was performed either on the ward or in the radiology department within between 1 and 18 hours of clinical suspicion of the formation of HO or DVT. In most patients, plain films and venography were performed either at the same visit or soon afterwards. A brief history and review of the events over the previous 48 hours was also taken. All scans were performed by one operator (RB) and follow up plain xray films and clinical assessments were made by all three authors. The US scan was considered abnormal if the normal lamellar pattern (Fig 1) was replaced by a chaotic disordered echopattern of the muscle fibres (Fig 2).

(a)

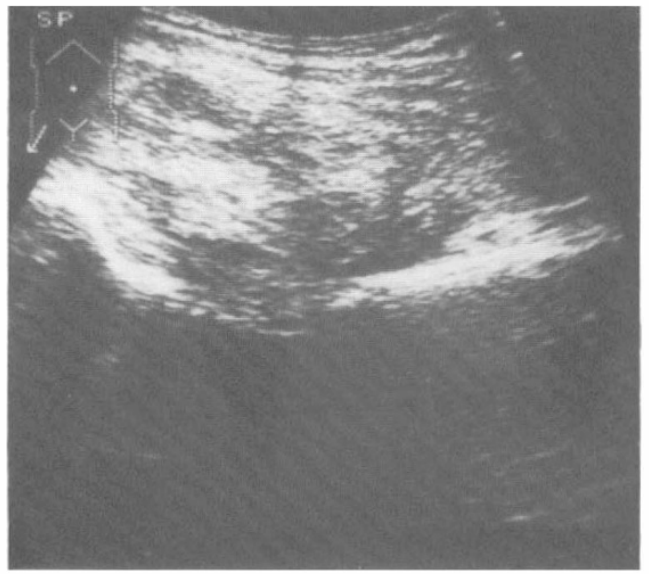

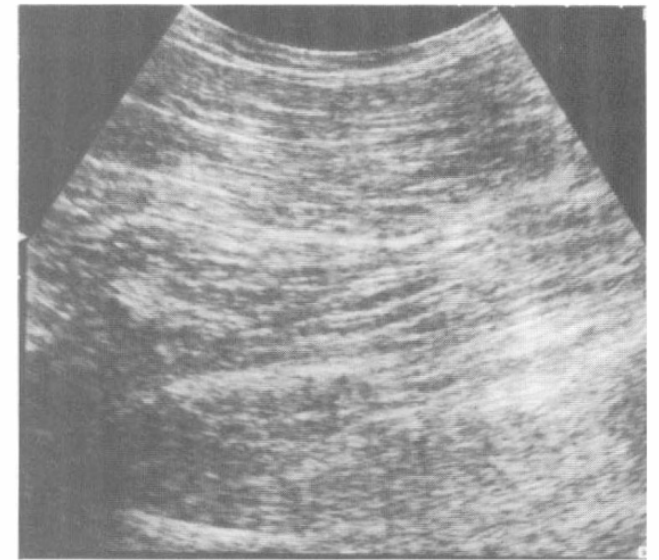

Figure 1 Ultrasound appearances of normal muscle in the thigh. Note the regular lamellar structure of the muscle fibres and intramuscular tissue planes.

\section{Results}

These are summarised in Table I. Twentyone patients (19 males, 2 females) with swollen legs were studied. Their ages ranged from 19 to 60 years with a mean of 29 years. Fourteen had complete tetraplegia and 7 had complete paraplegia. All but one had a possible traumatic predisposing factor (com-

(b)

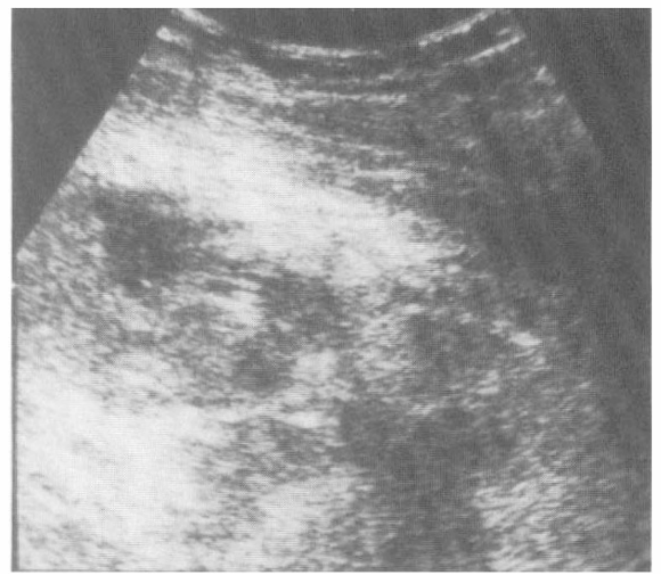

Figure 2 (a) Ultrasound appearances of an abnormal area in the thigh scanned on the day of clinical presentation. The regular lamellar pattern is lost and there is a chaotic, irregular echogenic pattern. HO was visible on the plain radiographs within 2 weeks. (b) Ultrasound appearances of the adductor region in a patient who had had adductor tenotomies 2 days previously. HO was visible on the plain films within 10 days. 
Table I Heterotopic ossification

\begin{tabular}{lcccccccc}
\hline Result & No $^{\mathrm{a}}$ & \multicolumn{1}{c}{ Age $^{\mathrm{b}}$} & $\begin{array}{c}\text { Weeks after } \\
\text { injury }\end{array}$ & Site & Anticoagulants & $\begin{array}{l}\text { Spasm/ } \\
\text { physio }\end{array}$ & Ultrasound \\
\hline Ossification & 14 & $19-49(29)$ & $8-30(13)$ & $\begin{array}{l}15 \text { hips } \\
1 \text { knee }\end{array}$ & $12 / 14$ & $\begin{array}{l}\text { All } \\
1 \text { post }\end{array}$ & Abnormal \\
? Ossification & 3 & $20-23$ & $12-22$ & hips & $3 / 3$ & $\begin{array}{l}\text { All } \\
\text { tenotomy }\end{array}$ & $\begin{array}{l}\text { Abnormal } \\
\text { (Rx EHDP) }\end{array}$ \\
No ossification & 4 & $19-60(31)$ & $2-8$ & $(4)$ & $\begin{array}{l}3 \text { hips } \\
1 \text { knee }\end{array}$ & $3 / 4$ & $3 / 4$ & $\begin{array}{l}\text { Normal } \\
1 \text { haematoma } \\
\text { around fix- } \\
\text { ation screw }\end{array}$ \\
\hline
\end{tabular}

a21 patients (19M, 2F).

${ }^{\mathrm{b}}$ Age range $19-60$, mean 29 yrs.

mencement of physiotherapy, transfer exercises or severe spasms, and in one patient recent adductor tenotomies), and all but 3 were fully anticoagulated.

Abnormal US findings were noted in 17 patients (a total of 18 thighs and one knee). Three of these patients started diphosphonate treatment immediately and received it for the remainder of the trial period. They did not develop HO. By 3 weeks however the remaining 14 showed evidence of $\mathrm{HO}$ on plain radiographs.

Normal findings apart from a small haematoma around a fracture fixation screw were observed in 4 patients. None of these subsequently developed $\mathrm{HO}$.

In the 4 patients who did develop $\mathrm{HO}$ the Doppler US showed a normal patent femoral vein with no evidence of DVT (Fig 3).

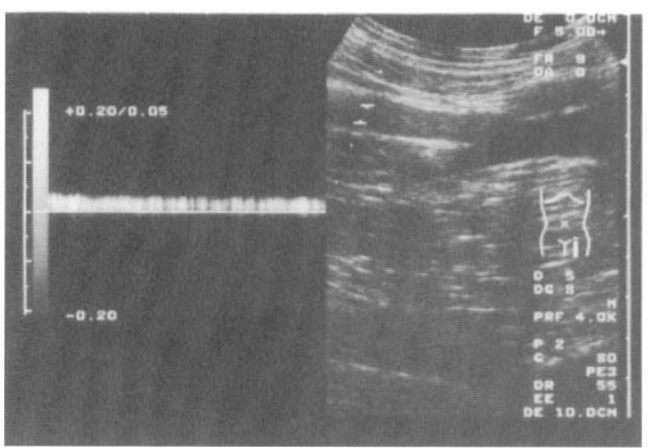

Figure 3 Doppler US trace of normal patent femoral vein at the groin.
All venograms were normal with no evidence of DVT, but they showed deviation of the femoral vein around a thigh mass (Fig 4).

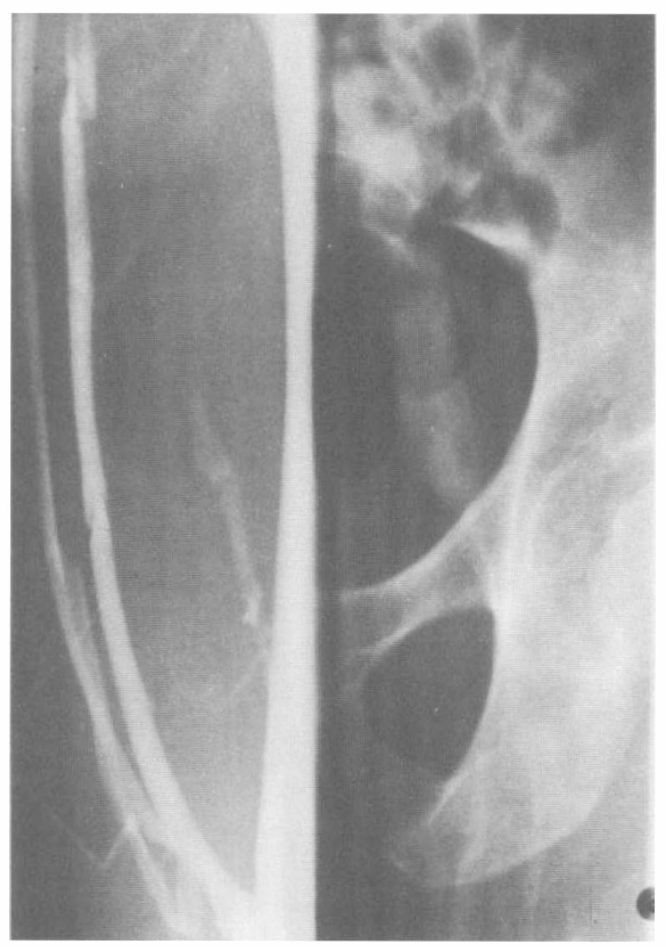

Figure 4 The opacified femoral vein can be seen taking a curved course around a soft tissue mass in the thigh. It is normally straight at this point. 


\section{Discussion}

For a series of review articles on HO, the reader is referred to Clinical Orthopaedics and Related Research 1991 263: 2-120.

Heterotopic ossification was first described in patients with spinal injuries by Dejerine in $1918 .{ }^{14}$ It was noted as a complication in patients with thoracolumbar fractures but surprisingly, not in those with cervical fractures, although it may be that very few tetraplegic patients survived long enough in that era. It has been noted in other conditions, particularly head injuries and severe burns, and it is postulated that some form of neural control exerted over the different mesenchymal cells present in the soft tissues is modified after the injury leading to a destabilisation of soft tissues. ${ }^{15}$ The most commonly affected areas are the hips and thighs, the knees and rarely the shoulders and elbows. The incidence is reported as between 1 and $50 \%$, the figures probably depending partly on the accuracy of reporting as well as reflecting different therapeutic techniques. It tends to occur 2-3 months after injury, predominantly in young men, and trauma, either from muscular spasm or physiotherapy, has been postulated as being implicated in the precipitating events although this is still controversial. ${ }^{16}$ The aetiology is still obscure and the pathological process is extremely complex. It probably involves an inflammatory cascade in genetically predisposed individuals that results in a dedifferentiation of mesenchymal and fibroblastic cells in the muscles and other soft tissues surrounding the joint and their conversion to osteoblasts. Over 7-10 days osteoid is laid down and calcification occurs with maturation to true bone over several weeks, when plain radiographs show calcification within the soft tissues. ${ }^{17-19}$

The presence of raised growth hormone levels, osteocalcin, osteoblast stimulating factors such as bone morphogenetic protein etc may have a role in research, but not clinical evaluation. ${ }^{20-22}$

Early diagnosis is currently difficult. Blood alkaline phosphatase levels rise before radiographic changes, but they are relatively nonspecific and are still raised 3 years after the initial event in approximately
$55 \%$ of patients. Plain radiographs may not show any abnormality for at least 10-14 days and although CT and MRI are more sensitive, they require transport of the patient to the radiology department and expensive investigation. Different histocompatibility surface antigens (HLA-BL18, -B27, -DW7 etc) have been found in variable percentages of the afflicted patients but they are time consuming and expensive to perform and do not have any place in the early diagnosis of $\mathrm{HO} .^{23}$

Animal studies and studies on patients undergoing total hip replacement arthroplasty (THR) have shown the benefit of nonsteroidal antiinflammatory drug administration $^{24}$ or local radiotherapy ${ }^{25}$ if it is commenced within 4-5 days of initial symptoms or surgery. Ethane hydroxydiphosphonate (EHDP) enjoyed a role in preventing ossification, but it was felt to be of little use if ossification had already started and in many patients in whom it kept ossification at bay, as soon as it was stopped the ossification proceeded unchecked. ${ }^{26-28}$ On theoretical grounds this is not unexpected as EHDP will only stop calcification of existing osteoid and therefore treatment should be aimed at preventing this osteoid formation in the first few days after the initiating event. Many centres now therefore use NSAIDs or radiotherapy. Prophylactic use in the group at risk has also been considered but is generally thought not to be warranted.

Currently the most favoured early test is the radionuclide bone scan with three phase assessment (arterial, venous and fixed bone phases). This is, however, an expensive investigation requiring transport to the radiology department, it uses ionising radiation and may not be positive in the first few days of the inflammatory process. It probably does have a role in the assessment of the maturity of bone formation if surgical excision is contemplated, although lack of change of activity on quantitative scans is not a good predictor of the likelihood of recurrence after resection. ${ }^{29,30}$

However, the clinical picture of a swollen leg, mildly elevated temperature with local heat, a woody swelling of the soft tissue and local erythema is also found in deep venous 
thrombosis, infection and tumours. While the latter two can generally be excluded clinically, the presence of a deep venous thrombosis must be excluded actively and therefore $\mathrm{HO}$ is effectively a diagnosis of exclusion. Venography is still currently the most widely accepted 'gold standard' for DVT diagnosis or exclusion in HO. Early studies often indicated a patent femoral vein deviating around a soft tissue mass. As high resolution real time ultrasound is the ideal method for investigating muscular tissues, US was performed on several patients and this revealed a very disordered muscular echotexture when compared with the normal side.

The study was therefore undertaken to assess the accuracy of this technique in determining abnormal focal muscle changes and later, using Doppler (without colour) the method was extended to look for deep venous thrombosis (Fig 3).

The study shows that US, which could be performed at the patient's bedside (Fig 5), was cheap, safe (using no ionising radiation) and reproducible. On several occasions the diagnosis on ultrasound was made within 20 minutes of the clinician being alerted to the changes in the leg and on two occasions changes were found in the opposite leg, which was clinically normal, but in which ossification subsequently developed. There was a very strong link between a traumatic precipitating event in the 48 hours preceding clinical change and the subsequent development of HO. This was predominantly a

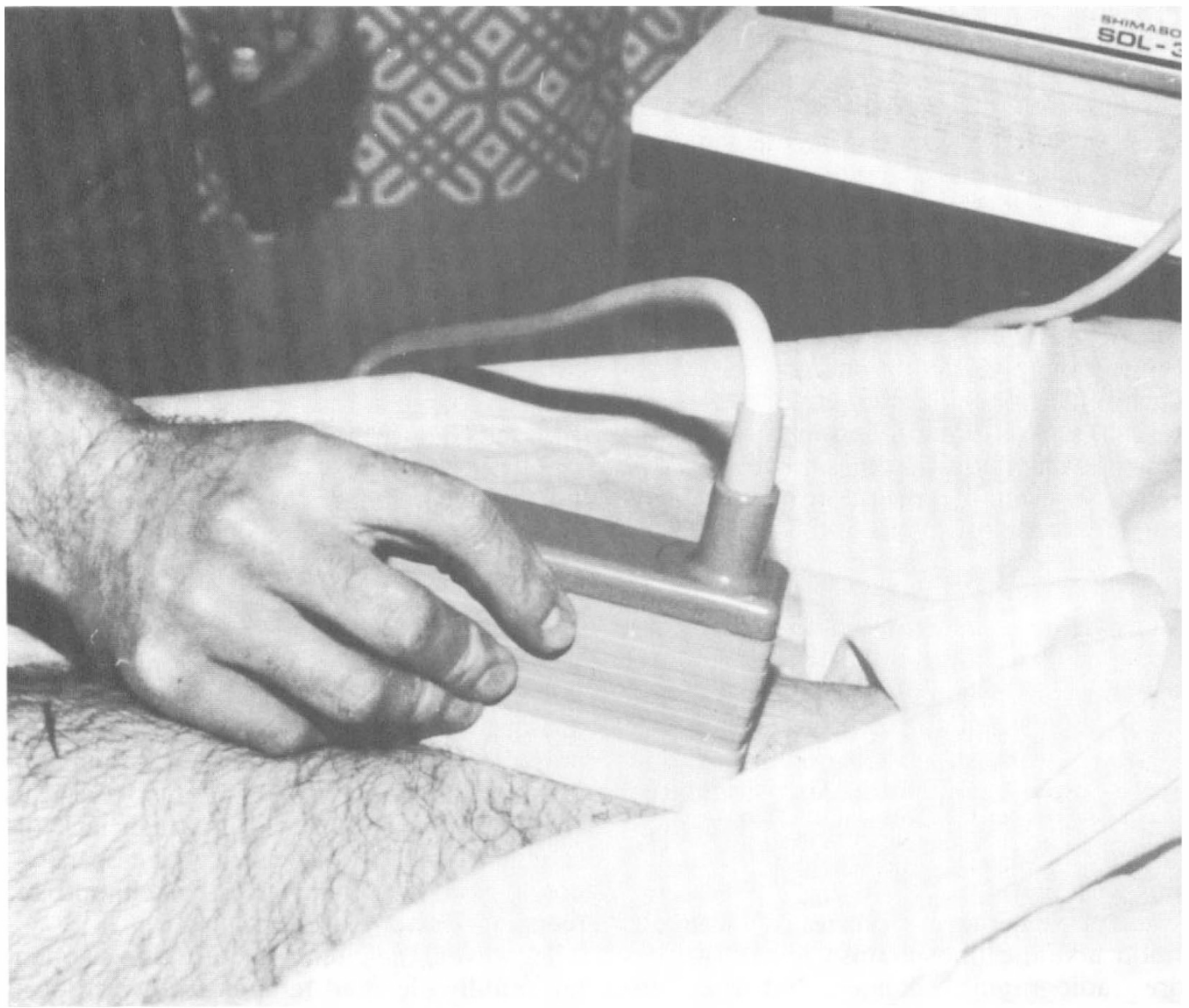

Figure 5 Ultrasound scanning in the patient's bed on the ward. 
change in physiotherapy or activity techniques (eg starting transfer training) or the presence of severe muscular spasm of the legs. In 3 patients, towards the end of the initial study, focal abnormalities were found and the patient was immediately started on EHDP as initial experience was already suggesting that US was an accurate assessment. HO was not evident subsequently, but one cannot tell whether this is the result of treatment or a false positive diagnosis. In 4 patients with mild degrees of swelling there was no focal abnormality and no subsequent $\mathrm{HO}$. In one patient a haematoma around a fixation screw of the knee was noted, but this did not later form ossification. In one patient who had had bilateral adductor tenotomies 3 days before presentation with swollen thighs and surface bruising, the changes suggested haematoma formation and marked ossification occurred on plain films within 2 weeks.

Very early in the study, initial appearances were interpreted as showing the presence of a haematoma and needle aspiration was attempted after anticoagulation had been reversed. As no blood was aspirated, needle biopsies were taken. Small tissue cores were obtained which showed osteoid formation and early bone formation with no calcification present, but Perl's staining showed the presence of haemosiderin laden macrophages which suggested the occurrence of microtrauma within the previous few weeks. These findings provide histological support for the theory that micro- trauma at least is involved in the aetiology of $\mathrm{HO}$ and will be presented in another paper.

\section{Conclusion}

The group of patients studied who developed $\mathrm{HO}$ were approximately $5 \%$ of the acute admissions to Stoke Mandeville Hospital over the examination period, being typical of those reported elsewhere with $\mathrm{HO}$ (predominantly young men $2-3$ months post injury). All had a history of previous recent trauma such as spasm, change in physiotherapy routine or surgery. In all positive cases ultrasound showed focal muscular abnormalities in areas which subsequently developed HO. DVT was excluded in all patients by venography and latterly by Doppler US.

Ultrasound is a very sensitive indicator and excluder of focal tissue abnormality and is sufficiently accurate to allow a rational start of therapy to prevent $\mathrm{HO}$.

It is the method of choice for early diagnosis of $\mathrm{HO}$ as it is cheap, safe (no ionising radiation) rapid, repeatable and portable to the patient's bedside. As it reliably diagnoses the main differential diagnosis, namely DVT, venography and other studies are therefore not necessary.

Ultrasound is potentially useful for a screening programme for patients at risk.

Limited biopsy experience suggests that trauma is involved in the aetiology of $\mathrm{HO}$.

\section{References}

1 Garland D (1991) A clinical perspective on common forms of acquired heterotopic ossification. Clin Orthop 263: 13-29.

2 Whittenberg RH, Peschke U, Botel U (1992) Heterotopic ossification after spinal cord injury. J Bone Joint Surg 74B: $215-218$.

3 Lal S, Hamilton B, Heinemann A, Betts HB (1989) Risk factors for heterotopic ossification in spinal cord injury. Arch Phys Med Rehabil 70: 387-390.

4 Biaro-Pagno P, Esclarin A, Arzoz T, Arroyo D, Lebarta C (1992) Incidence and risk factors in the appearance of heterotopic ossification in spinal cord injury. Paraplegia 30: 740-745.

5 Tibone J, Sakimura I, Nickel V, Hsu J (1978) Heterotopic ossification around the hip in spinal cord injury patients. J Bone Joint Surg 60A: 769-775.

6 Freed J, Hahn H, Menter R, Dillon T (1982) The use of the three phase bone scan in the early diagnosis of heterotopic ossification and in the evaluation of Didronel therapy. Paraplegia 20: 208-216.

7 Drane W (1983) Myositis ossificans and the three phase bone scan. AJR 142: 179-180.

8 Orzel J, Rudd T, Nelp W (1984) Heterotopic bone formation (myositis ossificans) and lower extremity swelling mimicking deep venous disease. J Nucl Med 25: 1105-7.

9 Fornage B, Touche D, Sigal P, Rifkin M (1983) Ultrasonography in the evaluation of muscular trauma. $J$ Ultrasound Med 2: 549-554. 
10 Fornage B, Eftekhari F (1989) Sonographic diagnosis of myositis ossificans. J Ultrasound Med 8: 463-6.

11 Thomas EA, Cassar-Pullicino V, McCall I (1991) The role of ultrasound in the early diagnosis and management of heterotopic bone formation. Clin Radiol 43: 190-196.

12 Cronan J, Dorfinan G, Scola F, Schepps B, Alexander J (1987) Deep venous thrombosis: ultrasound assessment using vein compression. Radiology 1162: 191-194.

13 Baxter G, McKechnie S, Duffy P (1990) Colour Doppler ultrasound in deep venous thrombosis; a comparison with venography. Clin Radiol 42: 32-36.

14 Dejerine A, Ceillier M (1918) Paraosteoarthropathies of paraplegic patients by spinal cord lesion. Translated in Clin Orthop 1991 263: 3-12.

15 Catz A, Suir D, Groswasser Z, Mendelson L, Solyi P (1992) Is the appearance of periarticular new bone formation related to local neurological disability? Paraplegia 30: 361-365.

16 Silver J (1969) Heterotopic ossification; a clinical study of its possible relation to trauma. Paraplegia 7: 220-230.

17 Smith R, Triffit J (1986) Bones in muscles: the problems of soft tissue ossification. $Q$ J Med 61(235): 985-990.

18 Smith R (1987) Head injury, fracture healing and callus. J Bone Joint Surg 69B(4): 518-520.

19 Ekelund A, Brosjo O, Nilsson O (1991) Experimental induction of heterotopic bone. Clin Orthop 263: 102-112.

20 Kurer M, Khoher M, Dandona P (1992) Human osteoblast stimulation by sera from paraplegic patients with heterotopic ossification. Paraplegia 30: 165-168.

21 Pietschmann P, Pils P, Woloszczuk W, Maerk R, Lessan D, Stipicic J (1992) Increased serum osteocalcin levels in patients with paraplegia. Paraplegia 30: 204-209.

22 Mohan S, Baylink D (1991) Bone growth factors. Clin Orthop 263: 30-48.

23 Hunter T, Dubo H, Hildahl C, Smith N, Schroeder M (1980) Histocompatability antigens in patients with spinal cord injury or cerebral damage complicated by heterotopic ossification. Rheumatol Rehabil 19, 97-99.

24 Kjaersgaard-Anderson P, Schmidt S (1991) Total hip arthroplasty, the role of anti-inflammatory medications in the prevention of heterotopic ossification. Clin Orthop 263: 78-86.

25 Ayers D, Pellegrini V, McCallisater Evarts C (1991) Prevention of heterotopic ossification in high-risk patients by radiation therapy. Clin Orthop 263: 87-93.

26 Stovers S, Hahn H, Miller J (1976) Disodium etidronate in the prevention of heterotopic ossification following spinal cord injury. Paraplegia 14: 146-156.

27 Finerman G, Stovers S (1981) Heterotopic ossification following hip replacement or spinal cord injury. Two clinical studies with EHDP. Metab Bone Dis Rel Res 4 \& 5: 337-342.

28 Chantraine A, Minaire P (1981) Paraosteoarthroplasties-a new theory and mode of treatment. Scand J Rehabil Med 13: 31-37.

29 Tanaka T, Rossier A, Hussey R, Ahnberg D, Treves S (1977) Quantitive assessment of PAO and its maturation serial radionuclide bone images. Radiology 123: 217-221.

30 Kim SW, Wu SY, Kim RC (1992) Computerised quantitative radionuclide assessment of heterotopic ossification in spinal cord injury patients. Paraplegia 30: 803-807. 